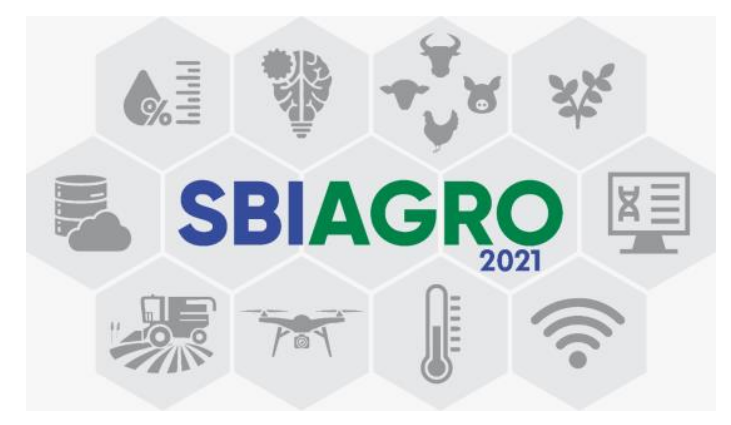

\title{
MIP² - um sistema de informação especializado em manejo integrado de pragas
}

\author{
Rodolfo Chagas Marinho Nascimento ${ }^{1}$, Monica Simão Giponi², Wellington \\ Moreira de Oliveira ${ }^{1}$, Vânia Maria Xavier ${ }^{2}$ \\ ${ }^{1}$ Departamento Acadêmico de Ciência da Computação, Instituto Federal de Educação, \\ Ciência e Tecnologia do Sudeste de Minas Gerais - Campus Rio Pomba, Rio Pomba, \\ Minas Gerais, Brasil \\ ${ }^{2}$ Departamento de Acadêmico de Agricultura e Ambiente, Instituto Federal de \\ Educação, Ciência e Tecnologia do Sudeste de Minas Gerais - Campus Rio Pomba, Rio \\ Pomba, Minas Gerais, Brasil \\ nsc.rodolfodgmail.com, monicagiponi@gmail.com,
wellington.moreiradifsudestemg.edu.br, vania.xavierdifsudestemg.edu.br
}

Resumo. O Manejo Integrado de Pragas (MIP) é uma ferramenta que contribui com a tomada de decisão quanto ao momento ideal para a adoção de métodos de controle nos cultivos agrícolas. No entanto, a falta de conhecimento técnico e a quantidade de etapas a serem realizadas, torna a adoção do MIP custosa e passivel de erros no recolhimento dos dados. Diante disso, o presente trabalho propõe um sistema que acompanha o agricultor na utilização do MIP em sistemas orgânicos de produção. Para o desenvolvimento deste sistema, identificaram-se os principais pontos para auxiliar nas etapas de implementação do MIP e a melhor forma de transferir esses conhecimentos aos usuários. Como resultado, o sistema apresenta diversos métodos de controle alternativos, informações para nortear o desenvolvimento das culturas e a correta identificação das pragas, além de gráficos que auxiliam no manejo dos cultivos.

Abstract. Integrated Pest Management (IPM) is a tool that contributes to decision making regarding the ideal time to adopt control methods in agricultural crops. However, the lack of technical knowledge and the number of steps to be carried out make the adoption of the IPM costly and minded to errors in data collection. Therefore, the present work proposes a system that accompanies the farmer in the use of IPM in organic production systems. For the development of this system, the main points to assist in the stages of implementation of the IPM and the best way to transfer this knowledge to 
users were identified. As a result, the system features several alternative control methods, information to guide the development of crops and the correct identification of pests, as well as graphics that help in crop management.

\section{Introdução}

A computação tem proporcionado avanços em diversos setores da sociedade e uma das grandes áreas beneficiadas é o setor agrícola. A integração computacional na agricultura ampliou-se desde a Revolução Verde, período entre 1960 e 1970 em que houve a difusão de tecnologias agrícolas que permitiram um aumento considerável na produção [Octaviano 2010]. Desde então, muitos agricultores vêm se adaptando a esta nova realidade para ampliar e melhorar seu sistema de produção.

Nos sistemas agrícolas, um dos grandes desafios enfrentados pelos produtores é o controle de pragas, que pela falta de manejo adequado pode causar grandes prejuízos. Atualmente, um modelo amplamente utilizado no controle de pragas em sistemas convencionais é o emprego de métodos químicos de controle, assim que observada a presença da praga nos cultivos. Ações desse tipo podem causar um desequilíbrio no agro ecossistema, além de não promover o controle adequado das pragas e elevar o custo de produção [Picanço, Gonring e Oliveira 2010]. A utilização de métodos de controle alternativos é pouco difundida e ainda apresenta entraves quanto a sua adoção. Dentre estes, destacam-se a escassez de conhecimentos sobre os métodos alternativos e a pouca transferência e divulgação dos mesmos aos agricultores [Campanhola e Bettiol 2003].

Uma metodologia utilizada para auxiliar os produtores no manejo das pragas é o Manejo Integrado de Pragas (MIP), um conjunto de técnicas que visa manter as pragas abaixo do nível de dano econômico [Gallo et al. 2002]. No entanto, sua correta adoção requer conhecimentos que na maioria das vezes o produtor não possui, relacionados ao diagnóstico da praga e a tomada de decisão, podendo dificultar e desestimular o agricultor a utilizar esta técnica. Diante dessas limitações, a utilização de um sistema de informação seria muito bem-vindo, devido aos benefícios gerados no armazenamento, organização e consulta de dados, facilitando a compreensão de todo processo e aplicação do MIP.

A crescente expansão da tecnologia e o advento dos aplicativos móveis tornou possível oferecer ferramentas de forma acessível. Sendo assim, atualmente, estão disponíveis aplicativos que contribuem desde a gerência agrícola até o manejo das culturas, como o aplicativo Aegro ${ }^{1}$ e o Doutor Milho ${ }^{2}$. No entanto, mesmo que, de acordo com Rodrigues (2004), a utilização de softwares para área de MIP seja muito promissora, a presença e utilização de sistemas que introduzem as técnicas e acompanham na realização do MIP ainda é ínfima. Prevalecendo softwares de gerência das propriedades e de informações técnicas, como os citados acima.

Diante da demanda de tecnologias acessíveis para o controle, acompanhamento e difusão de informações relacionados ao MIP, este trabalho apresenta um sistema que

\footnotetext{
${ }^{1} \mathrm{https} / /$ play.google.com/store/apps/details?id=com.aegro.aegroapp\&hl=pt_BR\&gl=US

${ }^{2}$ https://play.google.com/store/apps/details?id=br.embrapa.app.doutormilho\&hl=pt_BR\&gl=US
} 
auxilia na sua adoção e na utilização de métodos de controle considerados alternativos. Desenvolvido nas plataformas Web e mobile, o sistema visa guiar os produtores rurais na gerência de suas propriedades e na realização do manejo de pragas em sistemas de produção orgânica.

O presente trabalho foi estruturado da forma que segue. A Seção 2 descreve o contexto histórico do MIP no Brasil. A Seção 3 apresenta a metodologia e as etapas de desenvolvimento do sistema $\mathrm{MIP}^{2}$. Na Seção 4 é realizado um comparativo com aplicativos existentes atualmente. A Seção 5 lista trabalhos que se relacionam com o presente trabalho. Por fim, na Seção 6, são feitas as devidas ponderações, conclusões e discussões sobre trabalhos futuros.

\section{Manejo Integrado de Pragas}

Para podermos entender a necessidade de se adotar o MIP precisamos realizar uma análise do contexto histórico acerca da situação em que se encontra o manejo de pragas no Brasil. Atualmente, o controle de pragas em propriedades rurais é realizado, em sua maioria, através de aplicações de produtos químicos, visando extinguir a população de insetos no campo. Normalmente, não se utilizam cálculos ou constatações da população das pragas em campo, esse tipo de produto é utilizado assim que o inseto-praga é identificado em campo. Desta forma, gerando aplicações de agrotóxicos em excesso e sem uma forma expressiva de controle. Tal prática causa inúmeras consequências como impactos ambientais e riscos à saúde humana, tanto para quem consome quanto para quem faz a aplicação destes produtos. Apesar do controle químico aparentar ser uma prática moderna, essa assertiva não é verdadeira. Segundo Gazzoni (2012), logo após a Segunda Guerra Mundial houve uma grande expansão quanto à utilização de inseticidas organossintéticos. Devido a este fato, rapidamente surgiram os primeiros problemas relacionados ao seu uso excessivo. Nas décadas vindouras, os entomologistas desenvolveram técnicas com o objetivo de diminuir esse rápido avanço dos agrotóxicos. Dentre essas técnicas podem-se destacar o "controle supervisionado de insetos" e o "controle integrado", que visavam monitorar o inseto-praga, utilizando-se de pequenas doses de inseticidas para controlá-los concomitantemente com o uso do controle biológico [Gazzoni, 2012]. Além dessas premissas, era intrínseco realizar um levantamento para saber se a população das pragas havia atingido um nível em que o prejuízo causado fosse maior que custo das medidas de controle necessárias.

Objetivando diminuir a população de pragas e consequentemente os danos por elas causados, em conjunto com estudos e estratégias para atingir esse propósito, originou-se o que hoje chamamos Manejo Integrado de Pragas. Ao incorporar o conjunto de conceitos do MIP no sistema de produção seria possível garantir efeitos benéficos, social e ambientalmente. Porém, para integrar seus ideais aos cultivos é imprescindível o desenvolvimento de conjuntos tecnológicos que reforcem e auxiliem na aplicabilidade dessa metodologia, também se atendo à realidade dos cultivos em um futuro próximo. Assim, permitindo sua adoção em larga escala e expansão das técnicas do MIP. Além disso, podemos afirmar que apenas a tecnologia não seria suficiente para tornar o MIP totalmente difundido entre os produtores, também se fazem necessárias políticas públicas de encorajamento à aplicação do MIP e conscientização dos produtores quanto ao quadro de sustentabilidade na agricultura.

\section{Manejo Integrado de Pragas apoiado por Sistema de Informação}


Inicialmente, com objetivo de realizar uma análise geral da aplicabilidade do MIP, foi feito o acompanhamento de um experimento no setor de olericultura do IFSEMG campus Rio Pomba. A partir deste acompanhamento, foi possível destacar quais eram os pontos mais importantes para a adoção do MIP por um usuário com pouca ou nenhuma experiência. Além de informações imprescindíveis para realizar um manejo de pragas corretamente, é disponibilizado um acompanhamento aos produtores durante as fases do manejo. Além disso, o sistema auxilia no levantamento populacional das pragas, identificando o momento ideal de se realizar o manejo. Desta forma, ao implantar esta ferramenta na propriedade, o produtor garante que o método de controle só será utilizado quando necessário, e não de forma aleatória como é comum no método convencional.

\subsection{Engenharia de Requisitos}

Para realizar o desenvolvimento deste projeto foram seguidas algumas etapas, sendo a primeira delas o levantamento de requisitos em conjunto com envolvidos na área de Agroecologia. A partir dos requisitos foram definidas as funções do sistema e a disposição das telas. Através da criação do diagrama de casos de uso ${ }^{3}$ foi possível traçar o perfil dos usuários, tendo como base o acompanhamento em campo e as atividades exercidas pelos produtores. Considerando que o aplicativo deve auxiliar o produtor no monitoramento e na gestão de seu cultivo foram criadas duas classes de usuários: produtor e funcionário. Assim, é possível que o produtor adicione funcionários à sua propriedade e permite os funcionários a realizarem o recolhimento de dados nas culturas em que estão cadastrados.

Por meio do acompanhamento da execução manual do MIP, foram definidas quais as informações o sistema deveria organizar e gerenciar. Referente às pragas-chave e seus inimigos naturais é importante apresentar os dados que facilitem a identificação em campo e exemplifiquem qual tipo de dano é causado pelo inseto. Da mesma forma, as informações referentes às culturas acometidas foram disponibilizadas para auxiliar $o$ manejo do produtor e, consequentemente, diminuir a ocorrência das pragas.

Em consonância com o conteúdo apresentado, foram definidas as melhores formas de apresentar a realização das amostragens de praga. Visando ajudar usuários pouco experientes no manejo de pragas, adotou-se o plano de amostragem convencional, que se baseia na coleta de um número fixo de amostras por área [Gallo et al. 2002]. Desta forma, foram realizadas pesquisas bibliográficas de fichas amostrais das pragas e das culturas escolhidas, onde já está estabelecido o número fixo de amostras e a porcentagem de infestação necessária para iniciar a adoção dos métodos de controle. Sendo assim, com base nos dados coletados durante a realização do plano de amostragem, é realizado um cálculo que compara o nível populacional identificado com os níveis já estabelecidos pela literatura. Como resultado, o sistema informa se é necessário adotar um método de controle ou não para o talhão amostrado.

Caso seja necessário a aplicação de um método de controle, o sistema disponibiliza uma listagem com as opções recomendadas para a praga em questão. Assim que o usuário realiza estas etapas, os dados são salvos e podem ser acessados por ele posteriormente para análise da flutuação da população de pragas ou métodos de controle aplicados. Estes dados são disponibilizados em forma de relatórios e gráficos,

\footnotetext{
${ }^{3}$ https:/github.com/nscRodolfo/MIP-IniciacaoCientifica/tree/master/Documentação
} 
que podem ser armazenados no dispositivo móvel, assim o usuário poderá ter um melhor controle de seu cultivo. Os relatórios, dados do usuário e demais informações também podem ser acessadas pela plataforma Web.

\subsection{Modelo lógico}

Após o levantamento inicial de informações e técnicas necessárias, iniciou-se a segunda etapa do desenvolvimento com a criação de um protótipo com o objetivo de medir a aceitação dos usuários quanto ao sistema. Este protótipo foi disponibilizado para testes, realizados por estudantes de Agroecologia e envolvidos na área, o que facilitou a identificação de falhas no layout e usabilidade. Após a correção dos problemas de navegação e acesso às informações, iniciou-se a terceira etapa com a implementação do aplicativo móvel e incorporação dos resultados das pesquisas bibliográficas ao banco de dados. Para isso, fez-se necessária a criação do modelo lógico do banco de dados (Figura 1) e a implementação do mesmo, assim tornando possível salvar os dados na nuvem através de uma plataforma de host.

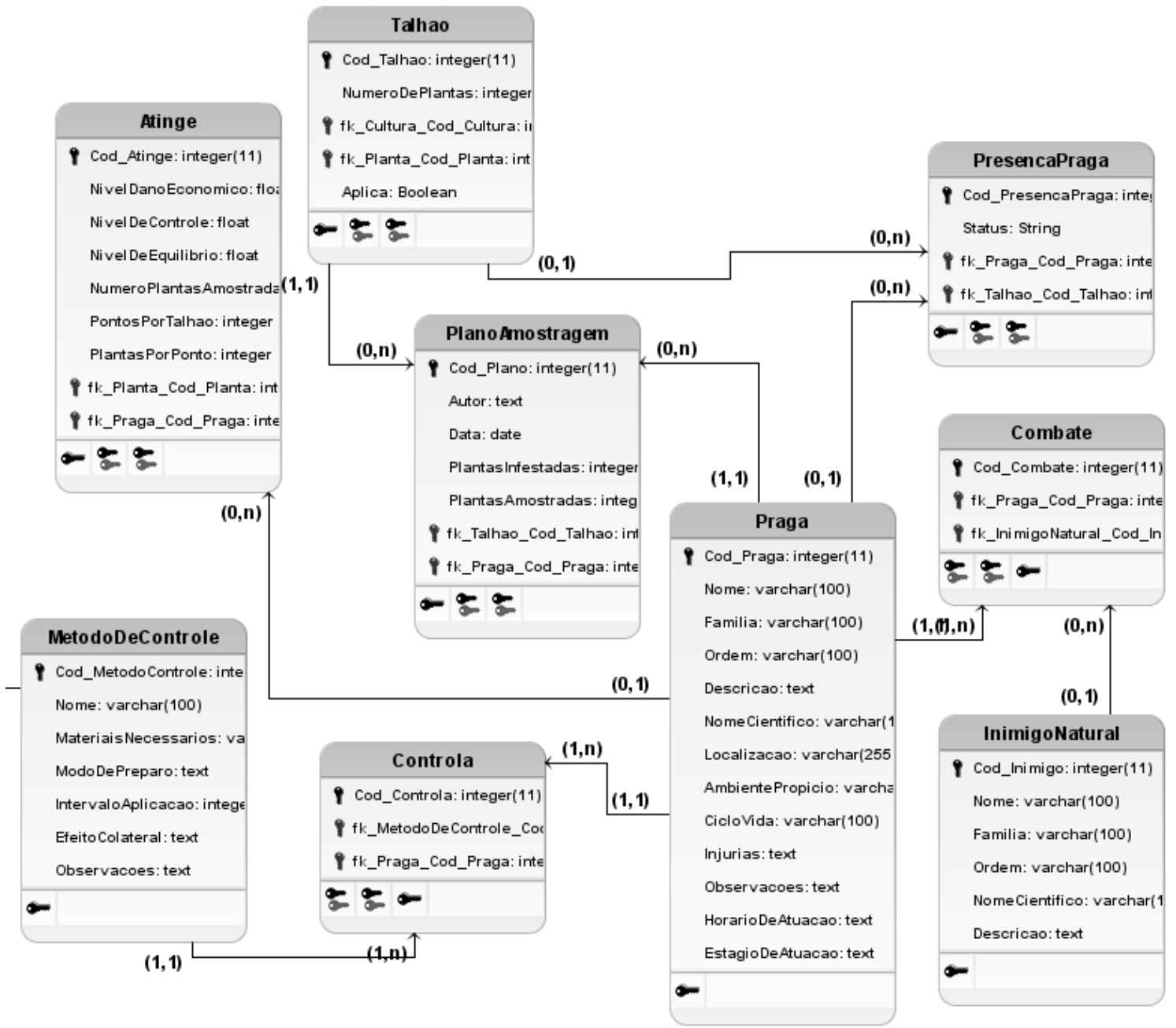

Figura 1. Parte do modelo lógico de banco de dados

A implementação foi realizada através do ambiente de desenvolvimento disponibilizado pela Google, Android Studio, que utiliza da linguagem JAVA para elaboração dos códigos e $X M L$ para desenvolvimento das interfaces. Assim que o desenvolvimento do aplicativo móvel foi finalizado, deu-se início à criação do módulo 
de suporte Web, feito na linguagem Hypertext Markup Language (HTML) com assistência de JavaScript e Hypertext Preprocessor (PHP) para comunicação com o Banco de Dados. Neste, definimos que seria criada uma forma mais simples de observação dos dados, deixando a parte de realização do monitoramento apenas para o aplicativo mobile. Desta forma, o módulo Web tem a função de visualização de informações e gerenciamento da propriedade. Assim que os dois módulos estavam finalizados foi realizada a interação entre ambas plataformas.

Mesmo com o desenvolvimento concluído ainda foi necessário aprimorar o aplicativo para suprir requisitos que se revelaram importantes durante a etapa de implementação do sistema. Dentre estes, destacou-se a necessidade de realizar o manejo de pragas de forma offline, já que a maioria das propriedades se encontram em zonas rurais, com pouco ou nenhum acesso à internet. Além disso, algumas áreas de cultivo são afastadas dos locais onde existe conexão wi-fi ou mesmo não possui rede móvel para conexão com a internet, isso acontece já que o acesso à internet é limitado e diversas vezes com alto custo em alguns locais da zona rural. Mesmo com este recurso é necessário que o agricultor consiga acesso à internet periodicamente para que o sistema possa realizar a sincronização dos dados. Caso o usuário não consiga se conectar à internet os dados estarão salvos apenas no dispositivo móvel.

\section{Resultados e discussão}

O sistema foi desenvolvido com o objetivo de auxiliar nas atividades de manejo de diversas culturas com importância comercial. Através dos dados disponibilizados, este software pretende capacitar o agricultor no entendimento e aplicação do MIP, mesmo que não tenha tido contato com essa prática. Para isso, guia o usuário no monitoramento das pragas (Figura 2(a)), orientando no caminhamento e na coleta das amostras. Além disso, contribui com a gerência da propriedade e permite observar os resultados da tomada de decisão, apresentados através de relatórios (Figura 2(b)). O $\mathrm{MIP}^{2}{ }^{4}$ foi publicado na Google Play Store, após adaptações na interface e códigos, tornando-se assim disponível para os usuários. Em conjunto com o aplicativo também foi disponibilizado um módulo Web que pode ser acessado pelo endereço "mip.software". 
$\equiv \quad M^{2} P^{2} \mid$ Pulgão verde

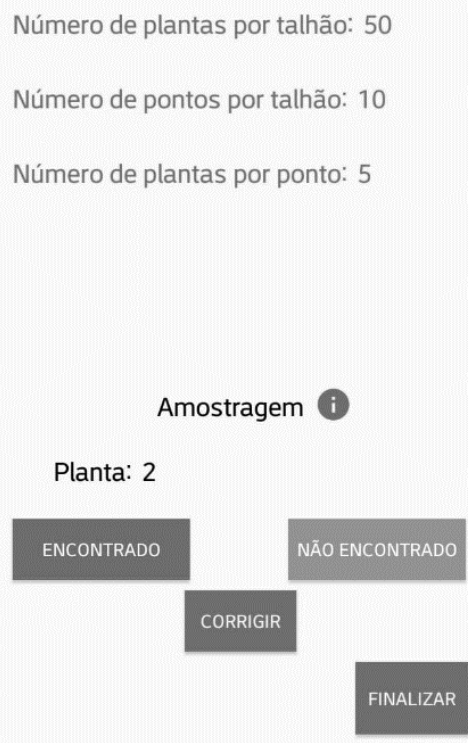

Figura 2(a) $\equiv \quad$ MIP2 | Berinjela x Mosca branca

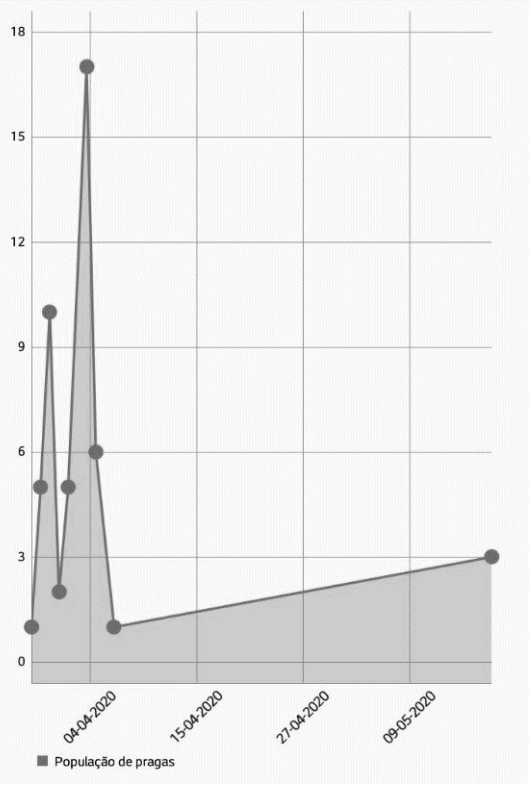

Figura 2(b)

Figura 2. Telas do aplicativo. Figura 2(a) - Plano de amostragem, Figura 2(b) Relatório de população de pragas.

A presença de sistemas especializados no manejo de pragas ainda é pequena quando se trata de dispositivos móveis. Os sistemas relacionados a este objetivo e que estão disponíveis na Google Play Store foram utilizados para um comparativo e avaliação do presente trabalho. De modo a comparar as principais funcionalidades disponíveis em cada aplicativo em relação ao $\mathrm{MIP}^{2}$, a Tabela 1 apresenta uma lista demarcando com um "X" a presença das mesmas em cada sistema.

Tabela 1. Variáveis a serem consideradas para aplicativos de manejo de pragas

\begin{tabular}{|c|c|c|c|c|c|}
\hline & $\mathrm{MIP}^{2}$ & $\begin{array}{l}\text { ADAMA } \\
\text { alvo }\end{array}$ & IZAgro & $\begin{array}{l}\text { Pragueiro } \\
\text { upcampo }\end{array}$ & $\begin{array}{l}\text { Guia } \\
\text { InNat }\end{array}$ \\
\hline Tutorial & $x$ & $X$ & & $x$ & $x$ \\
\hline Plataforma Web e Mobile & $\mathrm{X}$ & & & $\mathrm{X}$ & $x$ \\
\hline Multicultura & $x$ & $x$ & $X$ & $x$ & \\
\hline $\begin{array}{c}\text { Informações sobre as } \\
\text { culturas }\end{array}$ & $x$ & & & & \\
\hline $\begin{array}{l}\text { Apresenta as pragas } \\
\text { chave }\end{array}$ & $\mathrm{X}$ & $\mathrm{X}$ & $x$ & $x$ & \\
\hline $\begin{array}{c}\text { Apresenta os inimigos } \\
\text { naturais }\end{array}$ & $X$ & & & & $x$ \\
\hline Identificação de pragas & $x$ & $x$ & & & \\
\hline Métodos de controle & $x$ & $x$ & $x$ & $x$ & \\
\hline $\begin{array}{l}\text { Métodos de controle } \\
\text { alternativos }\end{array}$ & $\mathrm{X}$ & & & & \\
\hline
\end{tabular}




\begin{tabular}{|c|c|l|l|l|l|}
\hline $\begin{array}{c}\text { Forma de preparo e } \\
\text { intervalo de aplicação dos } \\
\text { métodos de controle }\end{array}$ & $\mathrm{X}$ & & & & \\
\hline Relatórios & $\mathrm{X}$ & & & $\mathrm{X}$ & \\
\hline $\begin{array}{c}\text { Auxilia na tomada de } \\
\text { decisão }\end{array}$ & $\mathrm{X}$ & & & & \\
\hline
\end{tabular}

Conforme os dados apresentados na tabela acima, podemos confirmar que o $\mathrm{MIP}^{2}$ é o software mais completo dentre os apresentados, tornando-se uma das mais completas soluções para aplicação dos conceitos do Manejo Integrado de Pragas. Um dos maiores diferenciais do sistema é a utilização apenas de métodos de controle alternativos, sendo vários deles recomendados e aprovados pelo Ministério da Agricultura, Pecuária e Abastecimento $\left(\mathrm{MAPA}^{5}\right)$. Desta forma, valorizando e incentivando o crescimento da agricultura orgânica brasileira. Os softwares Agricontrole e AgroPro Monitor, embora atuem na área de manejo de pragas, não foram avaliados neste comparativo por não apresentar uma versão disponível na Google Play Store ou por dificuldade no contato com os desenvolvedores.

\section{Trabalhos relacionados}

Atualmente encontramos diversos sistemas voltados para a área agrícola, isto ocorre, principalmente, segundo Galvão (2010), por conta da utilização de microeletrônica, biotecnologia e informática na produção agrícola. Apesar disso, na sua maioria, são sistemas que ajudam na gerência agrícola ou na divulgação de informações.

Como exemplo podemos citar o aplicativo Agricontrole [Kuhn 2018], que apresenta mapeamento da área, controle do tamanho dos cultivos, controle de rebanho e de estoque. Todas essas funcionalidades e informações são de suma importância para produtores rurais, porém não o auxiliam no desenvolvimento de técnicas agrícolas com o fim de melhorar o manejo das culturas.

Aplicativos que acompanham o produtor na aplicação das técnicas do MIP ainda estão em falta no mercado. Podemos encontrar alguns como, por exemplo o AgroPro Monitor $^{6}$ que auxilia, também, no levantamento de população de pragas. Porém, os sistemas que encontramos ainda são voltados para o manejo em produções convencionais, além de não apresentar e demonstrar as etapas para adotar o MIP.

Visando suprir essa necessidade o $\mathrm{MIP}^{2}$ foi criado, trazendo informações técnicas durante as etapas do processo de adoção do MIP. Além disso, com o intuito de fomentar a agricultura orgânica, apresenta apenas métodos de controle alternativos considerados orgânicos, trazendo os ingredientes, modo de preparo e intervalo de aplicação.

Este sistema acompanha o produtor de forma simples, explicando os processos e conduzindo-o durante a implantação e execução do MIP, com a possibilidade de verificar a eficiência dos métodos utilizados, além de monitorar a flutuação populacional por meio dos relatórios gerados.

\footnotetext{
${ }^{5} \mathrm{http}$ ///agrofit.agricultura.gov.br/agrofit_cons/principal_agrofit_cons

${ }^{6} \mathrm{https} / / /$ play.google.com/store/apps/details? $\mathrm{id}=$ com.agromonitor\&hl=pt_BR\&gl=US
} 


\section{Conclusões}

O desenvolvimento de ferramentas voltadas para a área agrícola é capaz de beneficiar os agricultores e contribuir com a difusão de técnicas que melhoram o setor produtivo. $\mathrm{O}$ sistema desenvolvido apresenta uma técnica específica que visa auxiliar e melhorar o manejo de pragas nas culturas cadastradas. Traz também, diversas informações sobre as pragas, culturas e métodos de controle que acrescentam ao produtor e contribuem com o manejo das culturas em um sistema de produção orgânica. Esta ferramenta também apoia a tomada de decisão quanto ao manejo adotado em cada talhão. Desta forma, por meio dos relatórios, o agricultor poderá verificar se o manejo para determinado talhão está contribuindo ou não com a baixa ocorrência de pragas em dado período, e assim realizar as possíveis adequações necessárias. Os relatórios apresentados, também podem colaborar com a rastreabilidade dos produtos quanto aos métodos de controle utilizados e auxiliar produtores que buscam uma certificação orgânica.

Como trabalho futuro pretende-se avaliar esta ferramenta com o público alvo para verificar sua utilidade e usabilidade, de modo a melhorar e adequar o software às demandas dos agricultores.

\section{Agradecimentos}

Esta pesquisa foi apoiada pelo IF Sudeste MG - Campus Rio Pomba e pelo Conselho Nacional de Desenvolvimento Científico e Tecnológico.

\section{Referências}

Campanhola, C. Bettiol, W. (2003) Métodos alternativos de controle fitossanitário. Jaguariúna: Embrapa Meio Ambiente.

Kuhn, F. (2018) "Agricontrole: aplicativo android para gerenciamento agrícola". Trabalho de Conclusão de Curso. Universidade Tecnológica Federal do Paraná.

Gallo, D. et al. (2002) Entomologia agrícola. Piracicaba: FEALQ.

Galvão, W. C. (2010) "Sistema WEB para gerenciamento de informações agrícolas". Dissertação de mestrado. Universidade Estadual de São Paulo.

Gazzoni, D. L. (2012) "Perspectivas do manejo de pragas", In: Soja: manejo integrado de insetos e outros Artrópodes-praga, Editado por Clara Beatriz Hoffmann-Campo, Beatriz Spalding Corrêa-Ferreira e Flavio Moscardi. Embrapa, Brasília.

Picanço, M. C. (2010) Manejo integrado de pragas. Viçosa: UFV.

Rodrigues, W. C. (2004). Utilização da Informática na Entomologia. Rev. Info Insetos p. 1-10.

Octaviano, C. (2010). Muito além da tecnologia: os impactos da Revolução Verde. ComCiência, p. 0-0. 